ing findings of nerve cells and glia cells. Our biochemical study on the same subje is under way.

Furthermore, assuming that when the head injury is caused by speed-factor, : many injuries are, this $-G$ factor have an important relation with the resultas injury. So we shall report the experimental findings.

\title{
51. A Study on Cardiopulmonary Function Following Head Injury
}

\author{
Nobuo MoriYasu, Kowten Satoh, Torao FujII, Yukio Morita, \\ Taro Watagami, Masakazu Honma, Saburo NaKamura, \\ and Hiroshi NAGAMINE
}

The First Department of Surgery, Nihon University, School of Medicine

Head injury was experimentally induced in dogs, and cardiopulmonary cir culation was observed by means of right heart catheterization and radiocardio. graphy (used ${ }^{51} \mathrm{Cr}$ ) up to one month after the injury.

Following the injury there was an increase in the pulmonary arterial anc capillary pressures and the total pulmonary vascular resistances and pulmonary arteriolar resistances. The mean pulmonary circulation time was prolonged after the injury, and a decrease in the cardiac output was noted. The pulmonary blood volume showed an increase. That is to say, these changes were most marked on about the 3rd day of the injury, decreased slightly in the first week and improved themselves considerably in the 2 nd week. The lungs assumed a congestive condition in the early stage of the injury.

There were no outstanding changes in the $\mathrm{pH}$ of peripheral arterial blood. The arterial oxygen saturation exhibited a decrease in the early stage of the injury, whereas there occurred an increase in the carbondioxide tension of arterial blood. An increase was perceived in the difference between arterial and mixed venous oxygen contents in the early stage of the injury alone.

From comparisons of these circulatory alterations with histological findings in the brain and lungs of the same dogs, congestion was seen histologically in the lungs until 2 weeks after the injury, when the pulmonary blood volume showed a high value. Especially in dogs with pulmonary edema and pneumonia, the mean pulmonary circulation time and the pulmonary blood volume exhibited significantly high levels.

Thus cardiopulmonary circulation undergoes great changes subsequent to head injury, but these changes can be considered to continue until about the 2 nd week. When the process of the recovery of the dogs from these changes is compared with 
the previously reported cerebral circulation, renal circulation, internal secretion, water electrolyte metabolism, etc., it is evident that cardiopulmonary circulation is in agreement with the latter in their periodical alterations, except for the delayed recovery of cerebral circulation and metabolism in severe cases.

\title{
52. Experimental Studies of Cerebral Circulation and Cerebral Metabolism in the Early Stage after Severe Head Injury
}

\author{
Kazuo KoJima, Setsuo Ono, Humio Nomura \\ Second Department of Surgery, Iwate Medical College \\ and Futoshi Mochiki \\ Second Department of Phisiology, Iwate Medical College
}

The changes of pathological physiology in the early stage after severe head injury in dog made by blank shots with air rifle were observed: especially the changes of oxygen availability and EEG in thalamus, blood pressure, blood flow, cerebro-spinal fluid pressure, $\mathrm{AO}_{2}, \mathrm{ERO}_{2}$ and $\mathrm{A}-\mathrm{VO}_{2}$ diff..

Oxygen availability and blood flow significantly increased in parallelism with blood pressure elevation and increase of cardiac output immediately after head injury, and decreased remarkably with falling the blood pressure.

One hour after head injury, oxygen availability and blood flow showed the lowest value. At this time slow activity in EEG appeared more frequently, and then oxygen availability and blood flow began to increase gradually, but three hours after injury not yet recovered to the level of before injury.

On the other hand, cerebral gas metabolism, namely $\mathrm{AO}_{2}, \mathrm{~A}-\mathrm{VO}_{2}$ and $\mathrm{ERO}_{2}$, reduced slightly three hours after injury.

As such changes of cerebral circulation and cerebral metabolism may be important ones which have close relations with changes in the early stage after severe head injury in man, we intend to analyse those changes experimentally hereafter. 